Review Article

\title{
The Heterogeneity of Neutrophil Recruitment in the Tumor Microenvironment and the Formation of Premetastatic Niches
}

\author{
Xin Xing $\mathbb{D}^{1}{ }^{1}$ Yongrui Bai $\mathbb{D}{ }^{2}$ and Jian Song $\mathbb{D}^{2,3}$ \\ ${ }^{1}$ Shanghai Fengxian District Central Hospital, No. 6600, Nanfeng Road, Shanghai, China 201499 \\ ${ }^{2}$ Department of Radiation Oncology, Renji Hospital, School of Medicine, Shanghai Jiao Tong University, Shanghai, China 200127 \\ ${ }^{3}$ Institute of Physiological Chemistry and Pathobiochemistry, University of Münster, 48149 Münster, Germany
}

Correspondence should be addressed to Yongrui Bai; baiyongruiz@163.com and Jian Song; songj@uni-muenster.de

Received 11 November 2020; Revised 26 December 2020; Accepted 9 February 2021; Published 19 February 2021

Academic Editor: Carlo Cavaliere

Copyright (C) 2021 Xin Xing et al. This is an open access article distributed under the Creative Commons Attribution License, which permits unrestricted use, distribution, and reproduction in any medium, provided the original work is properly cited.

The recruitment of neutrophil to the primary cancer has been shown to be steered by neoplastic cells or tumor-educated mesenchymal stromal cells and has a prometastatic effect. However, the neutrophil chemotaxis and their interaction with tumor cells in the distal metastasized tissues remain elusive. In this review, we discussed emerging research on the interaction between neutrophil recruitment and tumor metastasis, which is essential for studying tumor cell invasion and related immunotherapy.

\section{Main Text}

Metastasis is the basic malignant feature of cancer and the main cause of death in cancer patients. In distant metastasis, tumor cells interact with host organs to form a metastatic niche. Therefore, tumor metastasis is usually organ specific and, in many cases, is related to the systemic immune status. It is becoming increasingly clear that neutrophils associated with tumors play an important role in the occurrence and metastasis of many cancers [1]. However, according to the local microenvironment of neutrophils, they display great functional heterogeneity and multiple roles. Most neutrophil tumor researches focus on the early metastasis stage, during which neutrophils accelerate the spread of single tumor cells from the primary nidus and promote their infiltration into adjacent blood vessels. Here, we will put more emphasis on the metastasis-promoting effect of neutrophils on circulating tumor cells and how they promote tumor extravasation in distal blood vessels.

\section{Neutrophil Recruitment and Tumor Metastasis}

Tumor metastasis is a multistage process. When tumor cells metastasize, growth factors and cytokines secreted by stromal cells increase the ability of tumor cells to move, invade, and metastasize, causing epithelial-mesenchymal transition (EMT) of tumor cells. Activated tumor cells detach from the primary nidus and enter into the circulation, which is named as intravasation; if the tumor cells are not damaged by blood flow shear stress and immune cells in the blood vessels, they can adhere to the endothelial cells of the distal blood vessel, then penetrate the vessel wall and infiltrate into the distal tissues, which is named as extravasation. The extravasated tumor cells finally survive and proliferate in a new colonized environment [1-3].

2.1. Neutrophils and Tumor Intravasation. Tumor cell intravasation into the circulation is closely related to the angiogenesis of tumor vessels, as these vessels are much more 
accessible. Neutrophils also mediate the expression of various angiogenic factors such as vascular endothelial growth factor (VEGF) and matrix metalloproteinase- (MMP-) 9. Experiments have shown that MMP-9 produced by liver cancer-associated neutrophils could cleave the bound extracellular matrix (ECM) and benefit VEGF-A release, thereby promoting tumor angiogenesis [4]. In tumor transplantation models, the proangiogenic molecule Bv8 released by tumor-associated neutrophils can significantly promote tumor angiogenesis and tumor growth [5].

\subsection{Neutrophils and Circulating Tumor Cells. Intravasation} of tumors cells into the blood circulation provides the fast access to distal metastases. Although cancer cells may first invade the adjacent lymphatic vessels and accumulate in the draining lymph node, the majority of these cells will eventually enter the blood circulation and proceed further to the distant sites. Being a source of MMPs and growth factors, both neutrophils and platelets play a key role in the onset of tumor intravasation and help the tumor cells cross the endothelial barrier into the bloodstream [6]. More importantly, interaction with platelets and neutrophils can protect the circulating tumor cells from being eliminated by the bloodborne immune cells.

The tumor cells that enter circulation face multiple challenges, which include the detachment from the solid base and swimming in the flow with high shear stress [7]. Moreover, tumor cells in the circulation are under attack by the surveilling NK cells in the blood. Only the cells that extravasate at distant sites can survive. Killing circulating tumor cells or blocking tumor cell extravasation is a key line of defense against tumor metastasis, which, however, is often compromised by the recruited neutrophils and platelets.

In the circulation, tumor cells could be adhered by platelets. Platelet-derived transforming growth factor- $\beta$ (TGF- $\beta$ ) and platelet-derived growth factor (PDGF) could inhibit natural killer cell (NK) activity, and thus, platelet-covered tumor cells often escape the recognition and the lysis of NK cells. The tumor-activated platelets can also secret ATP, which binds the P2Y2 receptor expressed by endothelial cells and makes the vessel more permissive to the tumor extravasation [8]. Respectively, neutrophils have also been shown to suppress the immune response against circulating tumor cells. The presence of neutrophils constrains the activation of cytotoxic T cells and NK cells [9]. In addition, platelets could enhance the ability of neutrophil rolling on the endothelial cells and release chemokines, leading to further neutrophil recruitment. This effect enhances the extravasating rate of circulating tumor cells [10].

2.3. Neutrophils and Tumor Extravasation. Similar to the process of leukocyte extravasation, circulating tumor cell extravasation involves adhesion of tumor cells, transendothelial migration, and the migration across the vascular basement membrane into the metastasis sites.

2.3.1. Adhesion of Tumor Cells to the Endothelia. When the tumor cells move to the capillaries, they are physically blocked by the narrow capillaries. In the metastatic tissue, tumor cells that move on the endothelial layer can interact with the endothelial cells and upregulate the expression of E-selectin in resting endothelial cells. In the meantime, tumor cells express a variety of glycoprotein ligands of E-selectin, which promote the formation of initial adhesion $[11,12]$.

After initial adhesion, the firm adhesion of tumor cells is required for the subsequent transendothelial migration. Adhesion molecules such as integrins, MUC1, and CD44 are involved in the firm adhesion [13]. Integrins such as $\beta 1$ integrin, $\beta 4$ integrin, and $\alpha \mathrm{V} \beta 3$ integrin are shown to be upregulated, which facilitates tumor cell adhesion to the endothelial layer and their penetration through the endothelial and perivascular stroma [14]. In the metastasis of breast and colon cancer, MUC1 has been shown to interact with intercellular cell adhesion molecule-1 (ICAM-1), E-selectin, and galectin-3, promoting the firm adhesion of tumor cells to the adjacent endothelia [15]. After glycosylation, CD44 can also interact with the upregulated selectins and promote the endothelium adhesion and transendothelial migration of pancreatic and breast cancer cells [16].

2.3.2. Transendothelial Migration of Tumor Cells. Transendothelial migration is the process after firm adhesion, including paracellular and transcellular migration. Paracellular transendothelial migration refers to the migration of tumor cells through endothelial junctions. Studies have found that VEGF and TGF- $\beta$ produced by tumor cells and tumorassociated leukocytes destruct the vascular endothelium-cadherin- $\beta$-catenin complex, which weakens the endothelial junctions and promotes the paracellular transendothelial migration of tumor cells $[17,18]$. Angiopoietins secreted by breast cancer cells can lead to endothelial cell contraction and loosen the vascular barrier [18]. On the other hand, transcellular migration, the nonjunctional migration through endothelial cell, has been found in the cancer metastasis studies in vitro [19]. However, whether this occurs in vivo remains to be validated. Neutrophil extracellular traps (NETs) released by neutrophils play a crucial role in mediating tumor metastasis and recurrence. NETs are reticular structures released by neutrophil activation, which contain DNA, myeloperoxidase (MPO), and the proteins released by neutrophil degranulation and therefore can capture the invading microorganisms [20]. It has been shown that the circulating tumor cells could induce the release of NETs from their associated neutrophils, which in turn also capture the circulating tumor cells. The captured tumor cells cannot be killed, but will adhere to endothelial cells, survive from the lumen flow shear stress, and eventually extravasate [21, 22]. Neutrophils on its own could also adhere to the blood vessel or liver sinus and increase the incidence of tumor extravasation. More importantly, the neutrophils that extravasated into the premetastatic tissue accumulate and form the niche suitable for the incoming tumor cells [23].

After transmigration through the endothelial cell barrier, tumor cells invade the underlying basement membrane surrounding the blood vessel. $\beta 1$ integrin, focal adhesion kinase (FAK), and Rho GTPase are required for the 
invasion of tumor cells into the vascular basement membrane of mice. Eventually, tumor cells enter a new tissue to survive and proliferate [24]. During extravasation, tumor cells adapt their shape to the microenvironment by remodeling the actin assembly and actomyosin contraction, being round or elongated [25]. The round shape tends to overcome the shear forces of blood flow, while the cell in the elongated form can extend into the endothelial junctions, causing the endothelium contraction and promoting the transendothelial migration [26]. The physical properties and the chemical cues of the ECM determine the shape of tumor cells as well as their modes of migration.

\section{Heterogeneity of Cancer Metastasis}

The mechanisms of tumor cells extravasating into different tissues are likely to be different. Vessel permeability and the adhesion profiles of endothelial cell differ in diverse tissues, which correlate with the function of organs that they are located in. For example, the sinus structure of the liver or bone marrow could be permissive to the tumor cells, while the blood-brain barrier may require extra interactions to facilitate tumor cell infiltration into the brain. In addition, it has been shown that in the lung a special UDP/P2y6 axis promotes metastasis of melanoma by remodeling the premetastatic niche [27].

3.1. Lung. The lung is the most studied site of metastasis, in which researchers have shown that neutrophils acted as a major driver of metastatic colonization by breast cancer cells. Neutrophil transmigration in the lung is very different from transmigrations in the systemic microcirculation, where leukocyte extravasation occurs primarily through the postcapillary venules. Pulmonary extravasation of neutrophils occurs mainly in the alveolar capillaries. It is not an adhesion factor, but a biophysical factor in the pulmonary capillaries that blocks circulating neutrophils and directs them through endothelial cells [28]. In the case of tumor metastasis, neutrophils accumulate in the premetastatic lung microenvironment long before tumor cells arrive. Neutrophils can infiltrate into the lung and secrete proinflammatory factors such as leukotrienes to alter pulmonary vascular permeability and stimulate cell adhesions. On the other hand, tumor cells that expressed leukotriene receptors are recruited to the premetastatic lung. Blocking the synthesis of leukotrienes has shown strong inhibitions in the lung metastasis [23].

3.2. Liver. When migrating through the hepatic sinus, the agglomerated tumor cells can block the blood flow and the transient ischemia triggers an inflammatory reaction. Inflammation of the hepatic sinusoids can promote the adhesion of tumor cells to vascular endothelial cells, allowing tumor cells to escape the killing of Kupffer cells and NK cells. During this process, cell surface adhesion molecules E-selectin, vascular cell adhesion molecule-1 (VCAM-1), ICAM-1, and carcinogen embryonic antigen (CEA) play an important role. The number of neutrophils in the hepatic sinusoids is significantly increased during the early stage of metastasis, accompanied by an increased expression of CXCL1. Neutrophils in the hepatic sinusoids are often colocalized with metastatic tumor cells, and depletion of neutrophils significantly reduced tumor metastasis in the liver [29].

3.3. Central Nervous System. The blood vessel in the central nervous system is extremely tight and restrictive not only for migrating cells but also for soluble molecules. Microvessels of the central nervous system blood vessels are characterized by complex tight junctions between endothelial cells and the extra parenchymal basement membrane ensheathed by astrocyte endfeet, which collectively constitute the blood-brain barrier. However, tumor cells can cross the blood-brain barrier and survive and proliferate in the brain microenvironment. In contrast, most drugs can hardly reach the brain to kill tumor cells, which significantly increases the difficulty of the treatment of brain metastasis.

Brain metastasized tumor cells have strong interference on the structure and function of adjacent endothelial cells, which weakens the blood-brain barrier. In breast cancer, brain microvascular endothelial cells (BMEC) overexpress angiopoietin-2, which can alter the structure of tight junction protein ZO-1 and claudin-5, attenuating the integrity of blood-brain barrier [30]. Neuropeptide substance P secreted by breast cancer cells can activate BMEC to produce TNF- $\alpha$ and angiopoietin-2 and increase blood-brain barrier permeability [31]. Recent studies have found that both tumor cells and tumor-associated leukocytes are capable of producing cathepsin S, which cleaves the junctional adhesion molecule JAM-B. Drug inhibition of cathepsin S expression can reduce the occurrence of brain metastases [32]. Therefore, restoring the integrity of the blood-brain barrier might be a strategy to elude the brain metastasis.

\section{Intravital Imaging of Neutrophils in the Metastasis}

To investigate the metastasis, many approaches have been developed in the last decades, including both in vitro and in vivo studies. In vitro studies offer great opportunities in understanding the molecular mechanism and the signaling molecules involved in the tumor cell transendothelial migration. However, how these molecules work in the body remain elusive. Much of the in vivo knowledge on tumor cell migration into tissues has been traditionally derived from histological studies that lack assessment of the dynamics of cell behavior. To analyze the tumor extravasation in situ, high-resolution and rapid intravital microscopy (IVM) is favorable. IVM images dynamic cellular processes in live animals using phase-contrast or, more frequently, fluorescence microscopy. It has been developed over the last 10 years for the imaging of tumor cells and represents a powerful tool for studying cellular responses in time and space.

Cells tracking is commonly achieved by adoptive transfer of cells that are stained ex vivo. Cells can be labeled with membrane permeable dyes, such as TAMRA (5-(and-6)-carboxytetramethylrhodamin) and CFSE (5-(and-6)-carboxyfluoresceindiacetate, succinimidyl ester), lipophilic tracers 
such as carbocyanine dyes Dil and its derivatives that mark cell membranes, or with DNA labelling agents such as Hoechst 33342 to mark nuclei. Fluorescently labeled dextran can be injected intravenously into the animal, enabling delineation of the inner borders of blood vessels [33]. Intrastromal injection of labeled dextran can be used to delineate lymphatic vessels and reticular fiber networks within lymph nodes. More recent techniques focus on functional imaging, which allow investigation not only of cell dynamics in sit, but also real-time measurement of intracellular signaling pathways or metabolic pathways. One of the best examples of functional imaging is the incorporation of GFP-LifeAct, a 17-amino acid peptide derived from yeast that labels filamentous actin (F-actin) structures, into cells in vitro or in vivo. This permits real-time visualization of actin dynamics. To be able to track the metastasis, tumor cells can be labeled with photoconvertable protein, such as Kaede. Kaede is originally in green fluorescence but shifts to red by violet light, which allow the researchers to follow the same cells in both the primary and the metastasis locus [34].

\section{Clinical Studies of Tumor Extravasation}

Many clinical data indicate that the increase in the ratio of neutrophil-to-lymphocyte or the G-CSF level in peripheral blood often implicates a poor tumor prognosis or a poor chemotherapy effect. For example, the increased ratio of neutrophils to CD8+ T cells in surgically resected non-small-cell lung cancer tissues is associated with a high recurrence rate and shortened survival [35]. In the non-small-cell lung cancer, the increased peripheral blood neutrophil count is considered an independent poor prognostic factor. The ratio of serum MMP-9 level and the absolute neutrophil count can be used as a predictive biomarker for the efficacy of bevacizumab and platinum dual-target chemotherapy [36]. The relationship between the number of infiltrating neutrophils in tumor tissues and prognosis has been reported in different tumors. In recent years, there have also been related studies on mesenchymal tumors and neutrophils: neutrophil-tolymphocyte ratio is related to the progression of various soft tissue tumors such as osteosarcoma [37]. C3aR promotes the occurrence and development of malignant melanoma by inhibiting the effects of neutrophils and CD4+ T cells [38]. As the PD-1 antibody enters clinical trials and the efficacy of tumor immunotherapy has received much attention, neutrophil-to-lymphocyte ratio has been found to be related to the prognosis of patients treated with ipilimumab [39], indicating that the neutrophil count and related indicators can be used for phenotyping of tumor immunotherapy and evaluating the efficacy.

Based on the conceptual framework of tumor extravasation, many drugs have been designed or validated and most of them have so far focused on targeting the adhesion of tumor cells to endothelial cells. For example, both Seliciclib and Lovastatin have been shown to decrease the expression of E-selectin by endothelial cells and thus inhibit the tumor extravasation on the initial adhesion step [40-42]. JNK inhibitors have been shown to restrain extravasation of tumor cells by inhibiting both tumor cell adhesion and endo- thelial contraction. In the future, drug research on the signaling pathways and factors involved in the transmigration across the endothelial monolayer and the underlying basement membrane is needed [40].

\section{Concluding Remarks}

In this review, we have discussed the possibilities that neutrophils could be involved in the extravasation step of tumor metastasis. Under tumor conditions, neutrophils are polarized to produce the prometastatic molecules, which protect the circulating tumor cells from immune attacks and facilitate the tumor cells to extravasate blood vessels and into the distal organs. More specifically, various mechanisms are employed for neutrophils to adhere to vessels or sinuses and to penetrate the blood-brain barrier and pulmonary capillaries. With regard to the fact that each type of tumor cells has a preferential metastatic organ, we could assume that the local cues in those organs are different, which certainly requires further investigation. To study the dynamic process of tumor extravasation, we have to take the advantage of the advanced intravital imaging techniques and the emerging biotracking tools.

\section{Abbreviations \\ BMEC: Brain microvascular endothelial cells \\ CEA: Carcinogen embryonic antigen \\ EMT: Epithelial-mesenchymal transition \\ ECM: $\quad$ Extracellular matrix \\ F- actin: Filamentous actin \\ FAK: $\quad$ Focal adhesion kinase \\ ICAM-1: Intercellular cell adhesion molecule-1 \\ IVM: Intravital microscopy \\ MMP: Matrix metalloproteinase \\ MPO: Myeloperoxidase \\ MSC: $\quad$ Mesenchymal stromal cell \\ NK: $\quad$ Natural killer cell \\ NETs: Neutrophil extracellular traps \\ PDGF: $\quad$ Platelet-derived growth factor \\ TGF- $\beta$ : $\quad$ Transforming growth factor- $\beta$ \\ VCAM-1: Vascular cell adhesion molecule-1 \\ VEGF: Vascular endothelial growth factor.}

\section{Data Availability}

All the data in this study are available from the corresponding author upon reasonable request.

\section{Conflicts of Interest}

The authors declare that they have no conflicts of interest.

\section{Authors' Contributions}

We state that all the authors listed approved the manuscript.

\section{Acknowledgments}

This work is supported by grants from the National Natural Science Foundation of China (No. 31970745), the Innovative 
Medizinische Forschung foundation of Münster University of Germany (SO111713), Shanghai Municipal Health Commission (No. 201940506), and Fengxian District Science and Technology Commission Project (No. 20181802).

\section{References}

[1] P. Friedl and S. Alexander, "Cancer invasion and the microenvironment: plasticity and reciprocity," Cell, vol. 147, no. 5, pp. 992-1009, 2011.

[2] D. Gao, L. T. Vahdat, S. Wong, J. C. Chang, and V. Mittal, "Microenvironmental regulation of epithelial-mesenchymal transitions in cancer: figure 1," Cancer Research, vol. 72, no. 19 , pp. 4883-4889, 2012.

[3] D. X. Nguyen, P. D. Bos, and J. Massagué, "Metastasis: from dissemination to organ-specific colonization," Nature Reviews Cancer, vol. 9, no. 4, pp. 274-284, 2009.

[4] E. I. Deryugina, E. Zajac, A. Juncker-Jensen, T. A. Kupriyanova, L. Welter, and J. P. Quigley, "Tissue-infiltrating neutrophils constitute the major _in vivo_source of angiogenesisinducing MMP-9 in the tumor microenvironment," Neoplasia, vol. 16, no. 10, pp. 771-788, 2014.

[5] M. R. Galdiero, C. Garlanda, S. Jaillon, G. Marone, and A. Mantovani, "Tumor associated macrophages and neutrophils in tumor progression," Journal of Cellular Physiology, vol. 228, no. 7, pp. 1404-1412, 2013.

[6] A. Spiegel, M. W. Brooks, S. Houshyar et al., "Neutrophils suppress intraluminal NK cell-mediated tumor cell clearance and enhance extravasation of disseminated carcinoma cells," Cancer Discovery, vol. 6, no. 6, pp. 630-649, 2016.

[7] M. B. Headley, A. Bins, A. Nip et al., "Visualization of immediate immune responses to pioneer metastatic cells in the lung," Nature, vol. 531, no. 7595, pp. 513-517, 2016.

[8] D. Schumacher, B. Strilic, K. K. Sivaraj, N. Wettschureck, and S. Offermanns, "Platelet-derived nucleotides promote tumorcell transendothelial migration and metastasis via $\mathrm{P}_{2} \mathrm{Y}_{2}$ receptor," Cancer Cell, vol. 24, no. 1, pp. 130-137, 2013.

[9] S. B. Coffelt, K. Kersten, C. W. Doornebal et al., "IL-17-producing $\gamma \delta$ T cells and neutrophils conspire to promote breast cancer metastasis," Nature, vol. 522, no. 7556, pp. 345-348, 2015.

[10] M. Labelle, S. Begum, and R. O. Hynes, "Platelets guide the formation of early metastatic niches," Proceedings of the National Academy of Sciences of the United States of America, vol. 111, no. 30, pp. E3053-E3061, 2014.

[11] S. Hiratsuka, S. Goel, W. S. Kamoun et al., "Endothelial focal adhesion kinase mediates cancer cell homing to discrete regions of the lungs via E-selectin up-regulation," Proceedings of the National Academy of Sciences of the United States of America, vol. 108, no. 9, pp. 3725-3730, 2011.

[12] S. Köhler, S. Ullrich, U. Richter, and U. Schumacher, "E-/Pselectins and colon carcinoma metastasis: first _in vivo_evidence for their crucial role in a clinically relevant model of spontaneous metastasis formation in the lung," British Journal of Cancer, vol. 102, no. 3, pp. 602-609, 2010.

[13] T. Shibue and R. A. Weinberg, "Integrin 1-focal adhesion kinase signaling directs the proliferation of metastatic cancer cells disseminated in the lungs," Proceedings of the National Academy of Sciences of the United States of America, vol. 106, no. 25, pp. 10290-10295, 2009.
[14] S. R. Barthel, D. L. Hays, E. M. Yazawa et al., "Definition of molecular determinants of prostate cancer cell bone extravasation," Cancer Research, vol. 73, no. 2, pp. 942-952, 2013.

[15] H. Barrow, X. Guo, H. H. Wandall et al., "Serum galectin-2, -4, and -8 are greatly increased in colon and breast cancer patients and promote cancer cell adhesion to blood vascular endothelium," Clinical Cancer Research, vol. 17, no. 22, pp. 70357046, 2011.

[16] J. E. Draffin, S. McFarlane, A. Hill, P. G. Johnston, and D. J. J. Waugh, "CD44 potentiates the adherence of metastatic prostate and breast cancer cells to bone marrow endothelial cells," Cancer Research, vol. 64, no. 16, pp. 5702-5711, 2004.

[17] A. Hoeben, B. Landuyt, M. S. Highley, H. Wildiers, A. T. Van Oosterom, and E. A. De Bruijn, "Vascular endothelial growth factor and angiogenesis," Pharmacological Reviews, vol. 56, no. 4, pp. 549-580, 2004.

[18] D. Padua, X. H. F. Zhang, Q. Wang et al., "TGF $\beta$ primes breast tumors for lung metastasis seeding through angiopoietin- like 4," Cell, vol. 133, no. 1, pp. 66-77, 2008.

[19] S. Khuon, L. Liang, R. W. Dettman, P. H. S. Sporn, R. B. Wysolmerski, and T. L. Chew, "Myosin light chain kinase mediates transcellular intravasation of breast cancer cells through the underlying endothelial cells: a three-dimensional FRET study," Journal of Cell Science, vol. 123, no. 3, pp. 431440, 2010.

[20] N. Branzk and V. Papayannopoulos, "Molecular mechanisms regulating NETosis in infection and disease," Seminars in Immunopathology, vol. 35, no. 4, pp. 513-530, 2013.

[21] J. Cools-Lartigue, J. Spicer, B. McDonald et al., "Neutrophil extracellular traps sequester circulating tumor cells and promote metastasis," The Journal of clinical investigation., vol. 123, no. 8, pp. 3446-3458, 2013.

[22] J. Park, R. W. Wysocki, Z. Amoozgar et al., "Cancer cells induce metastasis-supporting neutrophil extracellular DNA traps," Science translational medicine, vol. 8, no. 361, p. 361ra138, 2016.

[23] S. K. Wculek and I. Malanchi, "Neutrophils support lung colonization of metastasis-initiating breast cancer cells," Nature, vol. 528, no. 7582, pp. 413-417, 2015.

[24] T. Shibue, M. W. Brooks, M. F. Inan, F. Reinhardt, and R. A. Weinberg, "The outgrowth of micrometastases is enabled by the formation of filopodium-like protrusions," Cancer Discovery, vol. 2, no. 8, pp. 706-721, 2012.

[25] V. Sanz-Moreno and C. J. Marshall, "The plasticity of cytoskeletal dynamics underlying neoplastic cell migration," Current Opinion in Cell Biology, vol. 22, no. 5, pp. 690-696, 2010.

[26] N. Reymond, J. H. Im, R. Garg et al., "Cdc42 promotes transendothelial migration of cancer cells through $\beta 1$ integrin," Journal of Cell Biology, vol. 199, no. 4, pp. 653-668, 2012.

[27] J. Qin, Z. Zhang, Z. Fu et al., “The UDP/P2y6 axis promotes lung metastasis of melanoma by remodeling the premetastatic niche," Cellular \& Molecular Immunology, vol. 17, no. 12, pp. 1269-1271, 2020.

[28] G. G. Van den Eynden, A. W. Majeed, M. Illemann et al., "The multifaceted role of the microenvironment in liver metastasis: biology and clinical implications," Cancer Research, vol. 73, no. 7, pp. 2031-2043, 2013.

[29] H. K. Avraham, S. Jiang, Y. Fu, H. Nakshatri, H. Ovadia, and S. Avraham, "Angiopoietin-2 mediates blood-brain barrier impairment and colonization of triple-negative breast cancer 
cells in brain," The Journal of Pathology, vol. 232, no. 3, pp. 369-381, 2014.

[30] P. L. Rodriguez, S. Jiang, Y. Fu, S. Avraham, and H. K. Avraham, "The proinflammatory peptide substance $\mathrm{P}$ promotes blood-brain barrier breaching by breast cancer cells through changes in microvascular endothelial cell tight junctions," International Journal of Cancer, vol. 134, no. 5, pp. 10341044, 2014.

[31] L. Sevenich, R. L. Bowman, S. D. Mason et al., "Analysis of tumour- and stroma-supplied proteolytic networks reveals a brain-metastasis-promoting role for cathepsin S," Nature Cell Biology, vol. 16, no. 9, pp. 876-888, 2014.

[32] K. Stoletov, H. Kato, E. Zardouzian et al., "Visualizing extravasation dynamics of metastatic tumor cells," Journal of Cell Science, vol. 123, no. 13, pp. 2332-2341, 2010.

[33] P. Steven, F. Bock, G. Huttmann, and C. Cursiefen, "Intravital two-photon microscopy of immune cell dynamics in corneal lymphatic vessels," PLoS One, vol. 6, no. 10, article e26253, 2011.

[34] T. Torcellan, H. R. Hampton, J. Bailey, M. Tomura, R. Brink, and T. Chtanova, "In vivo photolabeling of tumor-infiltrating cells reveals highly regulated egress of T-cell subsets from tumors," Proceedings of the National Academy of Sciences of the United States of America, vol. 114, no. 22, pp. 5677-5682, 2017.

[35] M. Ilie, V. Hofman, C. Ortholan et al., "Predictive clinical outcome of the intratumoral CD66b-positive neutrophil-to-CD8positive $\mathrm{T}$-cell ratio in patients with resectable nonsmall cell lung cancer," Cancer, vol. 118, no. 6, pp. 1726-1737, 2012.

[36] K. Hiura, A. Shiraishi, C. Suzuki et al., "MMP-9/ANC score as a predictive biomarker for efficacy of bevacizumab plus platinum doublet chemotherapy in patients with advanced or recurrent non-squamous non-small cell lung cancer," Cancer biomarkers : section A of Disease markers, vol. 15, no. 4, pp. 433-440, 2015.

[37] W. H. Smith, V. Gupta, E. G. Demicco, I. Iofin, and R. G. Maki, "(P035) the prognostic value of pretreatment peripheral blood neutrophil-to-lymphocyte ratio in patients with soft tissue sarcoma," Oncology, vol. 30, Supplement, 2016.

[38] J. A. Nabizadeh, H. D. Manthey, F. J. Steyn et al., "The complement $\mathrm{C} 3 \mathrm{a}$ receptor contributes to melanoma tumorigenesis by inhibiting neutrophil and CD4+ T cell responses," Journal of Immunology, vol. 196, no. 11, pp. 4783-4792, 2016.

[39] P. F. Ferrucci, P. A. Ascierto, J. Pigozzo et al., "Baseline neutrophils and derived neutrophil-to-lymphocyte ratio: prognostic relevance in metastatic melanoma patients receiving ipilimumab," Annals of oncology : official journal of the European Society for Medical Oncology, vol. 27, no. 4, pp. 732738, 2016.

[40] N. D. Ebelt, M. A. Cantrell, and C. L. Van Den Berg, "CJun $\mathrm{N}$-terminal kinases mediate a wide range of targets in the metastatic cascade," Genes and Cancer, vol. 4, no. 910, pp. 378-387, 2013.

[41] M. Hamalukic, J. Huelsenbeck, A. Schad, S. Wirtz, B. Kaina, and G. Fritz, "Rac1-regulated endothelial radiation response stimulates extravasation and metastasis that can be blocked by HMG-CoA reductase inhibitors," PLoS ONE, vol. 6, no. 10, 2011.

[42] N. Berberich, B. Uhl, J. Joore et al., "Roscovitine blocks leukocyte extravasation by inhibition of cyclin-dependent kinases 5 and 9," British Journal of Pharmacology, vol. 163, no. 5, pp. 1086-1098, 2011. 